\title{
The adverse impact, assessment and management of sarcopenia in liver transplantation candidates and recipients
}

\author{
Guoru Lan, Chunhua Yu, Yuguang Huang \\ Department of Anesthesiology, Peking Union Medical College Hospital, Chinese Academy of Medical Sciences, Beijing, China \\ Correspondence to: Chunhua Yu, MD. Peking Union Medical College Hospital, No.1 Shuaifuyuan, Dongcheng District, Beijing, China. \\ Email: yuchh@pumch.cn.
}

Submitted Apr 09, 2021. Accepted for publication Jun 29, 2021.

doi: $10.21037 / \mathrm{hbsn}-21-145$

View this article at: https://dx.doi.org/10.21037/hbsn-21-145

Sarcopenia is one of the common complications for cirrhotic patients on the waiting list of liver transplantation (LT), which has proven to be the best therapy for end-stage liver disease (ESLD). Some severe complications, including ascites, variceal bleeding, coagulation disorders and hepatic encephalopathy, were carefully managed by clinical physicians. Comparing with these complications, sarcopenia does not affect the mortality during hospitalization that much, even though it might have negative effects on patients' long-term quality of life (QOL) or even survival.

Sarcopenia denotes a progressive loss of muscle mass and function. Depending on the severity of hepatic dysfunction of ESLD patients, the prevalence of sarcopenia ranges from $10 \%$ to $70 \%$ (1). Patients with sarcopenia manifests a low skeletal muscle mass, low muscle strength and overaccumulation of lipid in muscle. The European Working Group on Sarcopenia in Older People (EWGSOP) proposed a consensus definition of sarcopenia in 2010 (2), which made the diagnosis of sarcopenia based on low muscle strength, plus low physical performance or low muscle quantity or quality. Later, in 2019, a revised version of operational definition was introduced by EWGSOP2 (3). In this new criterion, probable sarcopenia was identified by low muscle strength, while the diagnosis could be confirmed by low muscle quantity or quality on the basis of low muscle strength. Moreover, sarcopenia with low physical performance was considered to be severe.

\section{Adverse impact of sarcopenia on life quality and survival in ESLD patients and LT candidates}

The etiology of malnutrition and sarcopenia in ESLD patients and LT candidates is combined by several aspects. Dietary restriction, insufficient intestinal absorption, impairment of nutrient synthesis and continuous loss of protein are the mostly mentioned reasons of malnutrition. Moreover, ESLD patients are usually in a hypermetabolic state due to elevated proinflammatory factor levels. For example, increasing gluconeogenesis is one of the hypermetabolic progress, which leads to excess amino acids consumption and then muscle loss (4).

$\mathrm{Up}$ to now, there has been plenty of researches proving that sarcopenia was associated with negative clinical outcomes of LT. In recent years, several eminent published literatures caught our attention. In February 2021, Lee et al. published their research concerning the impact of preoperative muscle mass changes on the prognosis of deceased donor liver transplantation (DDLT) (5). They retrospectively studied the medical records and 6-month follow-up records of 56 patients who received DDLT. Muscle area, measured based on computed tomography (CT) scans, was normalized to height and is reported as skeletal muscle index (SMI). SMI decreased throughout preoperative period (mean preoperative $\Delta \mathrm{SMI}=-13.04 \%$, $\mathrm{P}<0.001)$. Moreover, for patients with major SMI decreasing $(\triangle \mathrm{SMI} \leq 30 \%)$, the mean survival time was significantly shorter than other patients $(\mathrm{P}=0.009)$, while preoperative decreasing of fat mass was not a prognostic factor. This research indicated that muscle mass continuously decreased during perioperative period of DDLT, and the severity of muscle loss was significantly associated with a reduction in survival after DDLT. Likewise, Kumar et al. revealed that LT recipients with sarcopenia had a higher incidence of postoperative sepsis, neurological complications, longer 
ICU stay and ventilator support (6). This prospective observational study of 115 LT recipients also revealed that low SMI was an independent predictor for early mortality.

Bot et al. studied the impact of muscle quantity and quality on ESLD patients who were on the waiting list of LT (7). Two hundred and sixty-one patients were retrospectively studied. Low SMI was found to be a significant predictor of an increased risk for waiting list mortality in LT candidates (Hazard Ratio $=2.580,95 \%$ CI: 1.055-6.308). Moreover, muscle attenuation (MA), a measure of muscle quality calculated with CT scan results, was also a statistically significant predictor of increased mortality in ESLD patients. In 2019, another study measured muscle mass with creatinine excretion. Higher muscle mass was associated with longer recipient survival at 1 year after orthotopic LT (8). Exercise has been proven related to improve muscle loss. A randomized trial of 151 LT recipients measuring QOL with Short Form 36 found that patients in exercise group had better QOL scores than usual care group (9). High-quality randomized trial evidence supporting the relationship between sarcopenia and QOL of LT recipient after transplantation is limited.

Aside from these three researches mentioned above, there has been more medical workers exploring the further associations between sarcopenia and ESLD. The adverse impacts of sarcopenia on pediatric LT candidates, infants' LT surgeries and liver re-transplantation were continuously studied. All these studies revealed that sarcopenia, manifesting as decrease of muscle quantity and quality, not only adversely influence the postoperative complications and survival of LT, but also associate with higher mortality for ESLD patients on the waiting list of LT.

\section{Assessment and management of sarcopenia in ESLD patients}

Evaluation methods of sarcopenia varies in different medical centers. The EWGSOP2 criteria suggests that sarcopenia could be assessed by the factors including muscle mass, muscle strength and physical performance (3). There have been several effective methods for each of these parameters.

Skeletal muscle mass is mostly evaluated by CT scans and magnetic resonance imaging (MRI), with SMI frequently used. L3 SMI is a typical parameter, which could be calculated with a transverse CT image from the L3 lumber spine vertebra. It took several major abdominal muscles into consideration, and could eliminate the influence of ascites. Psoas muscle area is a relatively new method, but could provide better accuracy than L3 SMI. Dual-energy $\mathrm{X}$-ray absorptiometry provides lower radiation exposure and is less expensive, but it's weakly concordant with CT scans due to failure to differentiate water from muscle (10). EWGSOP also recommend bioimpedance analysis because it's inexpensive, easy to use and accessible for bedside examinations. However, bioelectrical impedance analysis could be easily affected by fluid retention, food intake, BMI and some other factors. Mid-upper arm circumference and skinfold thickness could be applied in outpatient services, with relatively lower accuracy.

Muscle strength could be measured by handgrip strength. EWGSOP2 suggested that hand-grip strength $<27 \mathrm{~kg}$ for male and $<16 \mathrm{~kg}$ for female could be classified as muscle weakness (3). Peak expiratory flow could be applied for patients without lung diseases. Physical performance could be measured by 6-min walk test, preferred gait speed and stair climb power test.

Except these three parameters, biomarkers could also be considered as one part of general assessment. Alphafetoprotein (AFP) has been widely used in the evaluations of liver cancer. It's also been reported that AFP level $>100 \mathrm{mg} / \mathrm{dL}$ is associated with higher risk of sarcopenia in hepatocellular carcinoma patients undergoing LT (11). Prealbumin and albumin are common biological markers for nutritional assessments. In recent years, some other biomarkers have been advised for sarcopenia evaluation, such as procollagen type III $\mathrm{N}$-terminal peptide, circulating C-terminal agrin fragment, plasma extracellular heat shock protein 72 , and circulating skeletal muscle-specific troponin $\mathrm{T}$ (12). However, the associations between these biomarkers and sarcopenia of LT patients are yet to be discussed.

The management of sarcopenia for ESLD patients includes nutritional supplement and physical exercise. The theory that restricting protein intake might worsen the prognosis for ESLD patients is accepted by more and more physicians. For ESLD patients with sarcopenia, protein intake $>1.2 \mathrm{~g} / \mathrm{kg}$ of bodyweight per day is reasonable, with 25-30 g protein for each meal (13). Vitamin D and branched-chain amino acids are also recommended. Physical exercise is encouraged to optimize muscle mass and function, but the risk of variceal bleeding should be noticed for ESLD patients with portal hypertension. Severe cirrhotic patients might have a reduced exercise capacity, especially with severe complications like ascites. Postoperative nutritional management for LT patients should also be considered. After the transplantation, patients still require increased energy and protein intakes. For early post 
LT stage, $25-30 \mathrm{kcal} / \mathrm{kg}$ calories intake and $1.5-2.0 \mathrm{~g} / \mathrm{kg}$ protein intake are recommended. Early enteral nutrition or normal food intake is advisable (14). For long-term postoperative management, the risk of some metabolic disorders arterial hypertension, dyslipidemia and diabetes might increase, as results of excessive weight gain and immunosuppression. A multi-disciplinary and early monitoring of body weight should be encouraged. Appropriate exercise for LT recipients after transplantation is also advised. A recommended advice about exercise is to "start low, progress slowly, and be alert for symptoms". Exercise three times a week (20-30 min per session) has been proven to be effective for increasing skeletal muscle mass (15).

In conclusion, sarcopenia is a common complication for ESLD patients. It negatively influences the prognosis and mortality of LT recipients and ESLD patients on waiting list of LT. There has been variety of effective assessment methods for sarcopenia. Nutritional interventions and physical activities are efficient interventions.

\section{Acknowledgments}

Funding: None.

\section{Footnote}

Provenance and Peer Review: This article was a standard submission to the Hepatobiliary Surgery and Nutrition. The article has undergone external peer review.

Conflicts of Interest: All authors have completed the ICMJE uniform disclosure form (available at https://hbsn. amegroups.com/article/view/10.21037/hbsn-21-145/coif). The authors have no conflicts of interest to declare.

Ethical Statement: The authors are accountable for all aspects of the work in ensuring that questions related to the accuracy or integrity of any part of the work are appropriately investigated and resolved.

Open Access Statement: This is an Open Access article distributed in accordance with the Creative Commons Attribution-NonCommercial-NoDerivs 4.0 International License (CC BY-NC-ND 4.0), which permits the noncommercial replication and distribution of the article with the strict proviso that no changes or edits are made and the original work is properly cited (including links to both the formal publication through the relevant DOI and the license). See: https://creativecommons.org/licenses/by-nc-nd/4.0/.

\section{References}

1. Nam NH, Kaido T, Uemoto S. Assessment and significance of sarcopenia in liver transplantation. Clin Transplant 2019. doi: 10.1111/ctr.13741.

2. Cruz-Jentoft AJ, Baeyens JP, Bauer JM, et al. Sarcopenia: European consensus on definition and diagnosis: Report of the European Working Group on Sarcopenia in Older People. Age Ageing 2010;39:412-23.

3. Cruz-Jentoft AJ, Bahat G, Bauer J, et al. Sarcopenia: revised European consensus on definition and diagnosis. Age Ageing 2019;48:16-31.

4. Campos-Varela I, Gómez-Gavara C, Augustin S. Recommendations and guidance on nutritional supplementation in the liver transplant setting. Transplantation 2021. [Epub ahead of print]. doi:10.1097/ ТР.0000000000003736.

5. Lee J, Jeong WK, Kim JH, et al. Serial Observations of Muscle and Fat Mass as Prognostic Factors for Deceased Donor Liver Transplantation. Korean J Radiol 2021;22:189-97.

6. Kumar V, Benjamin J, Shasthry V, et al. Sarcopenia in Cirrhosis: Fallout on Liver Transplantation. J Clin Exp Hepatol 2020;10:467-76.

7. Bot D, Droop A, Lucassen CJ, et al. Both muscle quantity and quality are predictors of waiting list mortality in patients with end-stage liver disease. Clin Nutr ESPEN 2021;42:272-9.

8. Stam SP, Osté MCJ, Eisenga MF, et al. Posttransplant muscle mass measured by urinary creatinine excretion rate predicts long-term outcomes after liver transplantation. Am J Transplant 2019;19:540-50.

9. Krasnoff JB, Vintro AQ, Ascher NL, et al. A randomized trial of exercise and dietary counseling after liver transplantation. Am J Transplant 2006;6:1896-905.

10. Giusto M, Lattanzi B, Albanese C, et al. Sarcopenia in liver cirrhosis: the role of computed tomography scan for the assessment of muscle mass compared with dualenergy X-ray absorptiometry and anthropometry. Eur J Gastroenterol Hepatol 2015;27:328-34.

11. Acosta LF, Galuppo R, García CR, et al. Association Between Sarcopenia and AFP Level in Patients Undergoing Liver Transplantation for Hepatocellular Carcinoma. J Surg Res 2019;238:10-5.

12. Calvani R, Marini F, Cesari M, et al. Biomarkers for 
physical frailty and sarcopenia. Aging Clin Exp Res 2017;29:29-34.

13. Dasarathy S. Consilience in sarcopenia of cirrhosis. J Cachexia Sarcopenia Muscle 2012;3:225-37.

14. Anastácio LR, Davisson Correia MI. Nutrition therapy: Integral part of liver transplant care. World J Gastroenterol
2016;22:1513-22.

15. Kamimura H, Sato T, Natsui K, et al. Molecular Mechanisms and Treatment of Sarcopenia in Liver Disease: A Review of Current Knowledge. Int J Mol Sci 2021;22:1425.

Cite this article as: Lan G, Yu C, Huang Y. The adverse impact, assessment and management of sarcopenia in liver transplantation candidates and recipients. HepatoBiliary Surg Nutr 2021;10(4):575-578. doi: 10.21037/hbsn-21-145 